\title{
RESEARCH OF DYNAMICS OF DOUBLE HELICOIDAL VEHICLE IN GRANULAR MEDIA
}

\author{
Marcis Eimanis, Janis Auzins \\ Riga Technical University, Latvia \\ marcis.eimanis@gmail.com, auzinsjp@latnet.lv
}

\begin{abstract}
A new propulsion principle for underwater vehicles has been developed, inspired by microorganisms in the world of bacteria which use their rotating flagella to generate thrust force. The construction, consisting of two co-axial, left- and right-handed threads that rotate in opposite directions, which is termed double-helicoidal propulsion, and an elastic mid-section, which contains all communications and control mechanisms with a cardan joint, has successfully proven itself not only in water environment, but also shown the capability to perform as an amphibian. The prototype of an autonomous underwater vehicle created at the Institute of Mechanics of the Riga Technical University has shown good agreement with simulation results. Therefore, further studies are being conducted for a vehicle of this propulsion type, which include examination of its ability to move and maneuver in granular media. The main area of implementation for this propulsion type is remote or autonomous devices (robots). Such autonomous robots, which are able to move in granular media, for example, grain hoppers, could be successfully applied in gathering data and diagnostics of the hopper's internal environment. Equipping this kind of robot with humidity and thermal sensors would allow it to gather and show data in real-time, allowing prevention of potential problems. Discrete element modelling software EDEM was used to model interaction between granular media and moving objects with complicated forms. Simulation results confirm the ability of the patented vehicle to move in granular media that is not particularly dense. In the future it is planned to conduct real environment experiments with a prototype of the vehicle in a grain hopper.
\end{abstract}

Keywords: autonomous, vehicles, propulsion system, granular media, discrete element method.

\section{Introduction}

So far in nature and technology there have not been any notable vehicles or mechanisms discovered that would successfully move in granular media. Possibly it is due to impracticality of moving around in such areas, at least in comparison to solid ground or even water vehicles. The consumed energy while moving on solid ground is a lot lower than is required for achieving the same distance on loose ground. In turn, for water vehicles the force of inertia allows retaining movement even when the propulsion systems are shut down. In granular media under such conditions the surrounding resistance is too large, therefore all kinetic energy gets absorbed very quickly, but in case of, for example, car wheels and loose ground - the resistance of friction is too low to provide efficient traction. There is one example from the world of animals that can and does move in granular media, more precisely in a sand bed, and that is the sand swimmers (Plestiodonreynoldsi) (Figure 1). They use their body to make undulating motions to submerge under the sand surface.

Under certain conditions granular media has solid- or fluid-like properties. The connections between these properties are examined in the research, where with help of motion of robots on sand beds the researchers try to understand the balance between solid-like and fluid-like properties of granular beds [1]. In other words, granular media has properties of a fluid while its particles are in motion and properties of a solid - while they are static.

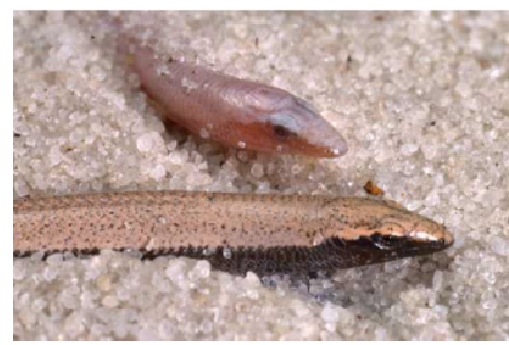

Fig. 1. Snapshot of sand swimmers (Plestiodonreynoldsi) emerging from granular bed. Figure credit: Alessandro Catenazzi

Since traditional propulsion types cannot efficiently move in granular environment, a newly designed and patented propulsion type is proposed. Initially it was built as an underwater propulsion system, but during the tests it showed good amphibian properties. The double-helicoidal (DH) 
propulsion principle consists of two right- and left-handed threads placed on the same longitudinal central axis. Allowing those threads to rotate in opposite directions produces thrust force, allowing the vehicle to "drill" through the surrounding media. In both computer and real environment experiments, the prototype showed good results, which confirmed the efficiency and potency of this propulsion type in an underwater environment. Therefore, further studies are now being conducted for a vehicle of this propulsion type, which include examination of its ability to interact with granular media.

Such DH propulsion principle can be applied to autonomous robots that would be able to explore and examine areas that are hard or even impossible to reach for human beings, for example, narrow caves in deep oceans, or huge grain hoppers and silos, to collect all the necessary data. If equipped with the necessary equipment, like humidity and thermal sensors, and using a wireless signal, it could show data in real-time.

\section{Granular media in modelling}

In nature granular media naturally is in a static state, but this state of rest is quite easy to disturb, similarly as for fluids. While the particles of the media are in a state of rest, the media has solid-like properties, but when the media starts to flow, it can be described as media with fluid-type properties. But what makes the fluids most different from granular media, even in micro scale, is that granular media consists of separated, non-contiguous particles. The easiest way to show this is by submerging an object in these environments -fluid will completely cover the entire surface of the object on which fluid forces can act on. But for an object that is submerged in granular media, every single particle that is in contact with the surface of the object will interact individually, concentrating its pressure on the surface in the point of contact.

In most situations loose media is not able to absolutely cover the surface of object, because there is always some free unfilled space between the particles. Here, though, it must be noted that for substances with finer particles, like sand or powder, the continuum model is used, where substance is like fluid, which consists of solid particle and gas or fluid combination [2]. In that way the granular media is considered as one whole, like fluid, not like a group with many separate particles. Such mixture is a two phase flow, in which the air and the solid material flow with their individual velocities, but are coupled through momentum exchanges resulting from pressure and viscous stresses. In high solid concentrations a strong coupling exists between the solid particles and the air, so the mixture can be approximately modeled as a single, composite fluid [3]. Although this continuum model has some limitations, it produces results with realistic features, as can be seen in Figure 2. This model also reproduces many characteristic features of granular flows that do not occur in pure fluids. For example, there can be regions of packed solid, where flow is prohibited entirely or is only exhibited along free surfaces having a slope exceeding the angle of repose [2].

a)

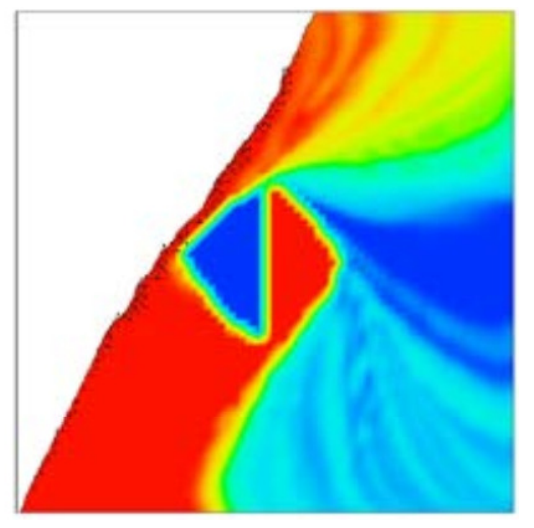

b)

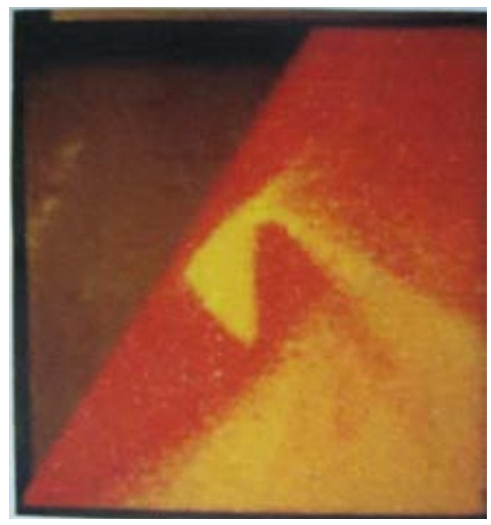

Fig. 2. Rotating rectangular mixer [2]: a - simulation data after 1.25 revolutions; $\mathrm{b}$ - experimental data after 1.25 revolutions

Other models do not use this continuum principle. There, the granular substance consists of separate particles, which can interact with one another. When modeling environment with such substances, contact models are used, for instance, the Hertz-Mindlin contact model [4], a simplified scheme of which is shown in Figure 3. This is a non-slip contact model, which is used to model 
contacts between particles. It is a spring-dash pot type nonlinear contact model, which is based on the Hertz-Mindlin contact theory. The normal force component is based on the Hertzian contact theory [5]. The tangential force model is based on Mindlin-Deresiewicz's work [6];[7]. Both normal and tangential forces have damping components where the damping coefficient is related to the coefficient of restitution, as described in [8]. The tangential friction force follows the Coulomb law of friction model as in, for example, [9]. The rolling friction is implemented as the contact independent directional constant torque model, see, for example, [10].

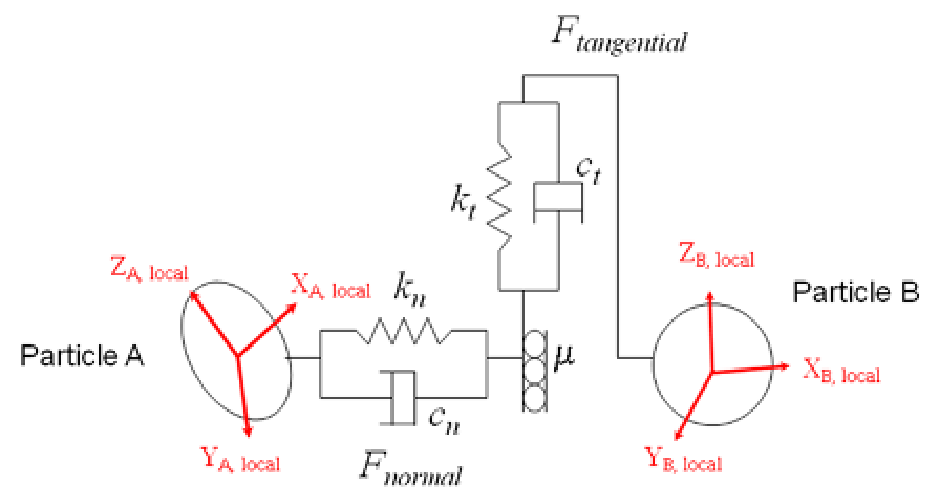

Fig. 3. Schematic of Simplified Hertz-Mindlin Contact Model: $k$ - particle stiffness; $c$-damping; $\mu$ - friction coefficient (credit: DEM Solutions $\odot$ )

Inelastic hard sphere (IHS) molecular dynamics with dissipative binary interactions are also applied. Here the interaction between the particles is governed by two major concepts: excluded volume and dissipation. Such models face the problem that during the contact moments finite time singularity state - inelastic collapse - may occur continuously between the particles. That is a pathology of idealized mathematical models[11]. Inelastic collapse manifests itself as infinite number of collisions in finite time. One way to avoid this is to apply the TC model [12]: if a particle has collision in a very small time window after its last collision, the collision is treated as elastic collision.

\section{Experiment}

The device, both the computer model and physical prototype, was built based on the aforementioned DH propulsion principle. It has been given the name durbis, which is derived from a combination of Latvian wordsmeaning"double-drill". For simulations of durbis in granular media the discrete element modeling software EDEM was used. The simulation model was built by analogy to what was used in building a simulation model for fluid.The existing threads of durbis were placed in a fixed state in cylindrical calculation domain giving them only one degree of freedom - the ability to rotate around the central axis, and the flow of granular media flows from the front. The granular substance flows in a horizontal direction with the assigned flow speed $v=0.6 \mathrm{~m} \cdot \mathrm{s}^{-1}$. Gravity is active in perpendicular direction. The bow and stern parts both rotate with the initial angular velocity. The diameter of the cylinder where durbis is placed is 1.4 times bigger than the thread diameter, which coincides with recommendations for models with fluids [13]. To simplify calculations, simple spherical particles were chosen, approximately the size of a barley grain. The properties of particles are as follows: Poisson's ratio $v=0.25$, solid density $\rho=600 \mathrm{~kg} \cdot \mathrm{m}^{-3}$ and shear modulus $G=4 \mathrm{E}+06 \mathrm{~Pa}$. The coefficient of restitution and coefficient of static friction values are kept as proposed by the Generic EDEM material model database, respectively 0.55 and 0.2 . For physics, for simplicity (but with sufficiently accurate results) the Hertz-Mindlin (no slip) contact model was used for both particle-particle and particle-geometry interactions.

The aim ofthe simulation was to find out whether durbis can move and gain any thrust force in granular media. During the experiments with durbis in fluid we gained the results, which showed that the angular velocities for the bow and stern parts were, respectively, $\omega_{b}=39 \mathrm{rad} \cdot \mathrm{s}^{-1}$ and $\omega_{s}=34.5 \mathrm{rad} \cdot \mathrm{s}^{-1}$ for the fluid flow speed of $v=0.6 \mathrm{~m} \cdot \mathrm{s}^{-1}$. That is why the initial flow speed of particles was set the same. Due to changes in the fluid flow conditions along the hulls of durbis, the differences in the bow and stern angular velocities are observable. But here, in granular media, such changes in 
the flow conditions are not anticipated. That is why in this situation the angular velocity for both the bow and stern parts is defined to be equal.

By obtaining variable particle flow rate, the moment was identified, when the particles in thread interspace and the rest of media have approximately the same flow speed. The image below, Figure 4, shows this moment in time. At this moment the angular velocity of the bow and stern parts was set to $\omega=35 \mathrm{rad} \cdot \mathrm{s}^{-1}$, and the flow speed is $0.4 \mathrm{~m} \cdot \mathrm{s}^{-1}$.

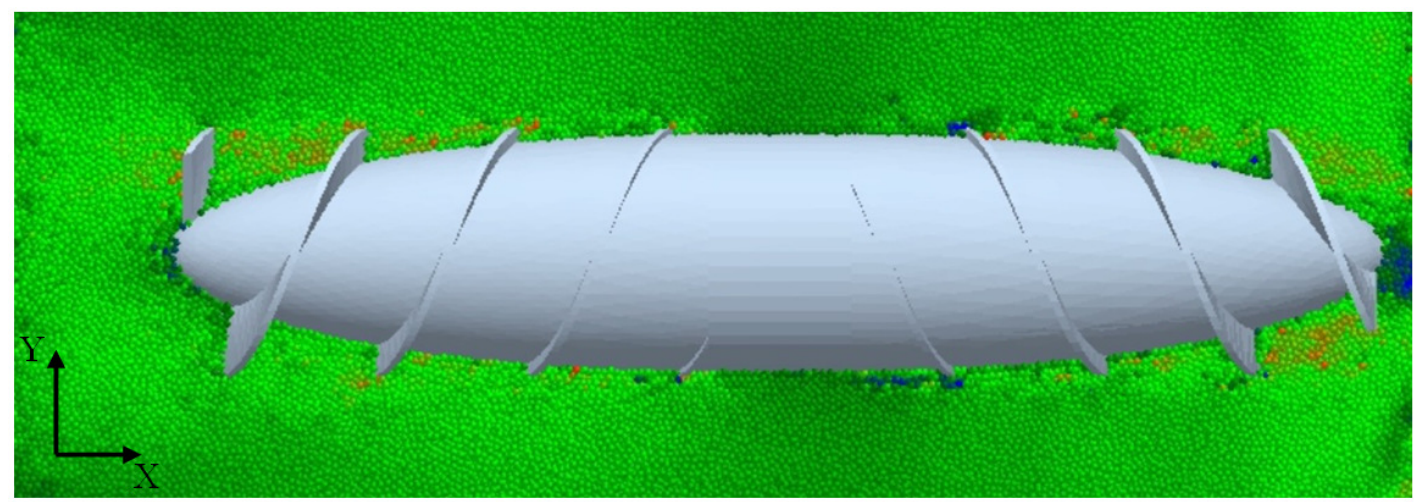

Fig. 4. Durbis in flowing particles

Here is a feature that also showed up during simulations with fluid. On the back of durbis there is a zone with, in this situation, particles standing almost still. In simulations with fluid at this zone the flow decreased its velocity close to zero, creating vortices due to turbulent flow state. This is one of the features that confirms the fluid-like characteristics of motion in granular media, or in other words similarity to fluid. It is also possible to see some decrease in the particle velocity on the front - this shows the ineffectiveness of the tip of the bow part. Making this tip slightly sharper would allow the particles to cross this zone faster.

Since it is not planned for durbis to move at relatively high speeds in loose media, the characteristic for slow motion of large intruders in granular media came into effect[14]: the drag force is independent of the velocity. Therefore, the drag force was not studied.

\section{Conclusions}

The results show that this type of propulsion system is able to gain thrust force in granular media, even with the existing configuration of threads. Better results could probably be achieved after applying optimisation for the tread and hull geometry.

During the experiments the hypothesis about the solid- and fluid-like properties of granular media was confirmed. The experiment showed some features that similarly appeared in the experiments with durbis in fluid.

Further research is required, including studies on how durbis is able to manoeuvre in loose media. This will require coupling EDEM software with multi-body dynamics software MSC Adams.

After optimizing the geometry of durbis and applying some corresponding sensors, like humidity and thermal sensors, it could autonomously move around in a grain hopper and gather all necessary data.

\section{Acknowledgements}

This research is funded by the Latvian Council of Science, project "Creation of design of experiments and metamodeling methods for optimization of dynamics of multibody 3D systems interacting with bulk solids and fluids", project No. lzp-2018/2-0281.

\section{References}

[1] Li C., Umbanhowarb P.B., Komsuogluc H. etc. Sensitive dependence of the motion of a legged robot on granular media. Proceeding of the National Academy of Sciences, March 3, 2009, USA, pp. 3029-3034. 
[2] Hirt C.W. A Continuum Model for Granular Flow. Flow Science Report 02-13, January 2013, Copyright Flow Science

[3] Hirt C.W.Scale Analysis of Two-Fluid Relative Velocity Equation: Evaluation of Drift-Flux Approximation.Flow Science, Inc. Technical Note 77, November 2007, Copyright Flow Science

[4] DEM Solutions, EDEM 2.6 Theory Reference Guide, 2014, p. 5.

[5] Hertz H. On the contact of elastic solids. Journal fur die Reine und AngewandteMathematik, Vol. 1882, issue 92,pp. 156-171.

[6] Mindlin R.D. Compliance of elastic bodies in contact. Journal of Applied Mechanics, Vol. 16,1949, pp. 259-268.

[7] Mindlin R.D., Deresiewicz H. Elastic Spheres in Contact under Varying Oblique Force. ASME, Journal of Applied Mechanics, Vol. 20, 1953, pp. 327-344.

[8] Tsuji Y. et al. Lagrangian numerical simulation of plug flow of cohesionless particles in a horizontal pipe. Powder Technology, Vol. 71(3), 1992, pp. 239-250.

[9] Cundall P.A.,Strack O.D.L.A discrete numerical model for granular assemblies.Geotechnique, Vol. 29, No. 1, 1979, pp. 47-65.

[10] Sakaguchi H. et al. Plugging of the Flow of Granular Materials during the Discharge from a Silo. International Journal of Modern Physics B, 07(09n10), 1993,pp. 1949-1963.

[11] McNamara S., Young W.R. Inelastic collapse in two dimensions. The American Physical Society, Vol. 50, no. 1, 1994, pp 28-31.

[12] Luding S., McNamara S. How to handle the inelastic collapseof a dissipative hard-sphere gas with the TC model, Granular Matter, Vol. 1, 1998, pp. 1-15.

[13] Watanabe T.,Kawamura T.,Takekoshi Y.,Maeda M.,Rhee S.H. Simulation of steady and unsteady cavitation on a marine propeller using a RANS CFD code. 5th Int. Symp. on Cavitation, November 1-4, 2003, Osaka, pp.1-4.

[14] Albert R., Pfeifer M. A., Barab'asi A. -L., Schiffer P. Slow drag in granular medium. Physical Review Letters, Vol. 82, 2005. 